\title{
FLORA AND HABITATS OF VENDICARI ISLET (ISOLA DI VENDICARI) IN SOUTH EAST SICILY
}

\author{
Pietro Minissale $^{1}$ \& Saverio Sciandrello ${ }^{2}$ \\ ${ }^{1}$ Department of Biological, Geological and Environmental Sciences, University of Catania, Italy \\ (e-mail: p.minissale@unict.it) \\ ${ }^{2}$ Centre for the Conservation and Management of Nature and Agroecosystems (CUTGANA), \\ University of Catania, Italy (e-mail: s.sciandrello@unict.it)
}

Minissale, P. \& Sciandrello, S.: Flora and habitats of Vendicari Islet (Isola di Vendicari) in South East Sicily. Nat. Croat., Vol. 26, No. 1., 1-16, Zagreb, 2017.

The vascular flora of 'Isola di Vendicari', a small islet (surface 4.45 hectares) located along the Ionian coast of south east Sicily, has been investigated. The surveyed taxa at specific and infraspecific level amount to 80 , of which 21 are new records from this islet. The life form spectrum of the current flora brings out the dominance of therophytes as well as a significant occurrence of hemicryptophytes. The plant communities colonizing the surface of the islet have been investigated from floristic, phytosociological and ecological points of view. The vegetation is represented mainly by psammophilous and halophilous aspects. As regards the conservation area policy, the islet falls within the Nature Reserve of Vendicari, which is also a SCI of the Natura 2000 network, showing its remarkable importance for the biodiversity conservation.

Key words: Sicily, Islet, vascular plants, phytosociology, biodiversity, conservation

Minissale, P. \& Sciandrello, S.: Flora i staništa otočića Vendicari (Isola di Vendicari) na jugoistočnoj Siciliji. Nat. Croat., Vol. 26, No. 1., 1-16, Zagreb, 2017.

Istraživana je vaskularna flora malog otočića 'Isola di Vendicari' (površina 4,45 ha) koji se nalazi uz jonske obale jugoistočne Sicilije. Utvrđeno je 80 vrsta i nižih svojti, od toga je 21 nova za otočić. U spektru životnih oblika prevladavaju terofiti, uz značajan udio hemikriptofita. Biljne zajednice na otočiću analizirane su s florističkog, fitocenološkog i ekološkog gledišta. Vegetacija je uglavnom psamofitska halofitska. S aspekta zaštite, otočić je dio Rezervata prirode Vendicari, nalazi se unutar područja Natura 2000 ekološke mreže, što ukazuje na važnost potrebe očuvanja bioraznolikosti.

Ključne riječi: Sicilija, otočić, vaskularna flora, fitocenologija, biološka raznolikost, očuvanje

\section{INTRODUCTION}

All over the Mediterranean Basin the flora of small and very small islands has long been a subject of interest not only for the sake of pure floristic knowledge but also for the understanding of the mechanisms of the colonization of these isolated micro-territories in relation to the flora of the mainland (see e. g. Arrigoni \& Bocchieri, 1996; Pandža, 2002; Bogdanović \& Mitić, 2003; Panitsa et al., 2006; Lo Cascio \& Pasta, 2012; Iliadou et al., 2014; PAsta et al. 2014; PANDŽa \& Milović, 2015; Jasprica et al., 2015; Fois et al., 2016; SCIANDRELLO et al., 2017).

The islet of Vendicari is located in the Ionian Sea near the south eastern coast of Sicily $\left(36^{\circ} 47^{\prime} 29^{\prime \prime} \mathrm{N} ; 15^{\circ} 6^{\prime} 18^{\prime \prime} \mathrm{E}\right)$; in Italian maps it is called »Isola di Vendicari «, but it is a very little island of $4.45 \mathrm{ha}$. This islet is uninhabited and landing is forbidden except for research purposes. In the last century from 1917 to 1943 it was seasonally inhabited for tuna fishing in support of the nearby tuna fishery (MALANDRINO, 2010), which was su- 
ccessively destroyed. Previously Houel (1785) wrote about this islet, on which he landed and made a drawing of the ancient ruins existing at that time.

On the islet, since 2010 the Audouin's Gull [(Larus audouinii (Payraudeau, 1826)] has nested and this is the only known site in Sicily (IENTILE et al., 2016). This species is included in the Annex I of »Bird Directive« 2009/147/CE. It states that special conservation measures must be provided with regard to the habitat, to ensure survival and reproduction of the listed species.

The vascular flora was investigated for the first time by Alво (1959), who visited this islet in 1909 and 1917, publishing his research many years later. The flora of the coast facing the islet, corresponding to Vendicari Reserve was recently studied by Minissale \& Sciandrello (2010) without taking the islet into account. Therefore, the aim of this paper is to fill this gap and update the floristic after the passage of a century, with some information on the plant communities that occur and the main drivers of the establishment of plants.

\section{STUDY AREA}

Vendicari Islet falls within the Vendicari protected area, one of the most interesting parts of Sicily, since it is representative of a remarkable variety of environments that have

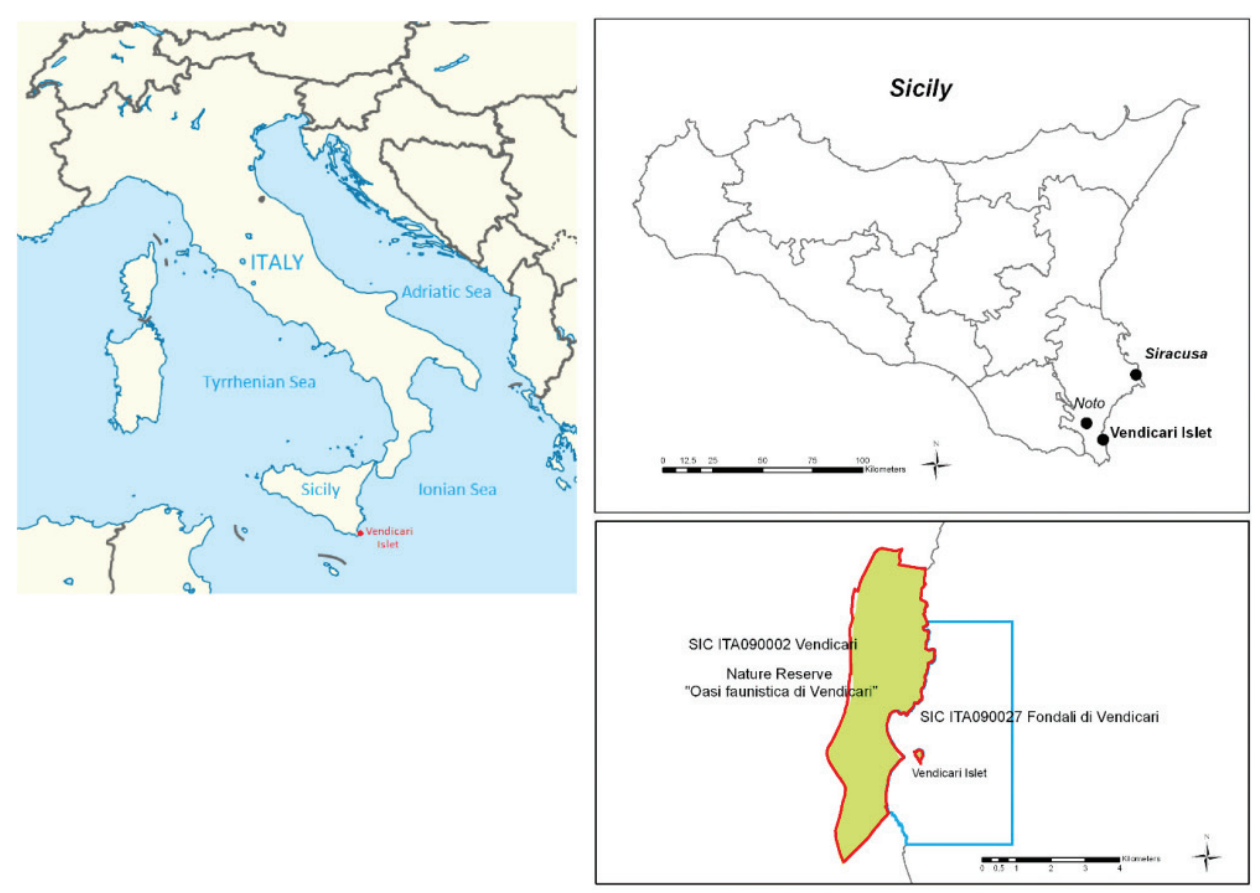

Fig. 1. Geographic location of Vendicari Islet (Isola di Vendicari). A. Location of the islet in Mediterranean Sea. Image from https://commons.wikimedia.org/wiki/File:Mediterranean_ Sea_location_map.svg, modified. B. Location of the islet in Sicily. C. The island and the mainland are a Nature Reserve named »Oasi Faunistica di Vendicari« [Faunal Oasis of Vendicari]. They are also included within the Site of Community Importance ITA090002 »Vendicari«, while the seabed belong to the SCI ITA090027 »Fondali di Vendicari«. 
largely disappeared or are now very degraded in the rest of the Sicilian territory. The reserve was established by the Regional Decree 03/14/1984 and lies entirely in the municipality of Noto, a town near Syracuse, in South-East Sicily (Fig. 1). The protected area extends over 1,512 ha: 575 ha in the A zone, having maximum protection, and 937 ha in the B zone or pre-reserve for agriculture and tourist activities-accommodation. The reserve is also a Site of Community Importance (SCI) of the Natura 2000 network under the name »ITA090002 Vendicari« as is the seabed, called »ITA090027 Fondali di Vendicari «. It is also part of the Special Protection Area (SPA) »ITA090029 Pantani della Sicilia sud-orientale, Morghella, Marzamemi, Punta Pilieri e Vendicari«.

The islet falls within the A zone and has a length of $320 \mathrm{~m}$ and a width of $255 \mathrm{~m}$ with an altitude of $2.5 \mathrm{~m}$. It is $445 \mathrm{~m}$ distant from the coast (data taken from Google Earth satellite photos 2016) with a seabed from 0 to $5 \mathrm{~m}$ deep.

Vendicari Islet is located in the eastern coastal sector of the Hyblaean Plateau, which is the emerged part of a gently deformed segment of the African continental margin (Dewey et al., 1989). In particular, the Vendicari coastal belt is characterized by sloping rocks mixed with sandy dunes, which are inwards often replaced by large brackish lagoons (Pantano Sichilli, Pantano Roveto, Pantano Piccolo and Pantano Grande). The sedimentary sequence is composed of terrains from Pliocene to Quaternary age. The lower level consists of the Trubi Formation, made of white marls of Pliocene age (LENTINI et al., 1996). Usually, there are deposits of massive yellow sandstones of Pliocene age above the white marls. Sometimes, at the top of this sequence there are white to yellowish organic calcarenitic and calciruditic deposits. In the islet these last two levels outcrop, partially covered by recent sands. All the area is affected by intense karstification, which accelerates the erosion of the whole rocky coast (Pirrotta \& Barbano, 2011; Gracia et al., 2014).

The Vendicari area is affected by a Mediterranean bioclimate, with mild winters and hot and dry summers. For these analyses we refer to the weather station of Cozzo Spadaro near Portopalo (ZAmpino et al., 1997). In this locality the average annual rainfall does not reach $400 \mathrm{~mm}$ per year, with highs of over $60 \mathrm{~mm}$ in October, December and January, and values close to zero in the summer months (June to August). The average annual temperature is $18.2^{\circ} \mathrm{C}$. Overall the bio-climate of the area can be defined lower thermo-Mediterranean, lower dry, according to BAzAN et al. (2015).

\section{MATERIALS AND METHODS}

Field trips in the Vendicari Islet were carried out in May 2012. Floristic sample covered the whole surface of the islet and the exsiccata (preserved in the Herbarium of University of Catania - CAT) were studied using the Italian floras (FIORI, 1923-29; PIGNATTI, 1982) and some taxonomic revisions or monographs, while the nomenclature follows Giardina et al. (2007). The surveyed taxa are listed (Tab. 1), in alphabetical order. For each taxon, collectors, notes, life form and chorological element are reported. The life form classification follows the Raunkiaer system as proposed by PignatTi (1982).

Vegetation was studied according to the Braun-Blanquet approach (BRAUN-BLANQU$\mathrm{ET}, 1964)$. The phytosociological analysis of the vegetation allowed the detection of some plant communities and the understanding of their ecological characteristics with considerations of their syntaxonomical position according to BRULlo et al. (2002) and BIONDI et al. (2014). In order to draw a habitat and vegetation map, aerial photos, dated 2007, were used and elaborated with ArcGis 10.3. Besides, in Tab. 2 the Corine Biotopes codes 


\section{Tab. 1. Floristic list of Vendicari Islet}

Legend: Coll. Ac = collected by ALвo (1959), confirmed; $\mathrm{n}=$ new record for the islet.

Notes: $\mathrm{nr}=$ not recorded now; $\mathrm{NV}=$ new to the Vendicari Nature Reserve flora; $\mathrm{pm}=$ probably misidentified.

Life Forms: $\mathrm{T}$ = therophyte; $\mathrm{H}=$ hemicriptophyte, $\mathrm{G}=$ Geophyte, $\mathrm{Ch}=$ Chamaephyte, $\mathrm{NP}=$ nano-phanerophyte, $\mathrm{P}=$ phanerophyte; scap = scapose; caesp = caespitose (tufted); bulb = bulbouse; rhiz = rhizomatous; succ = succulent; suffr $=$ suffruticose; frut $=$ fruticose; bien = biennial; ros = rosulate .

Chorotypes: End. $=$ Endemic; Med. = Circum-Mediterranean; C Med. = Centre Mediterranean; W Med. $=$ West Mediterranean; E Med. $=$ East Mediterranean; S Med. = South Mediterranean; Med. Trop. $=$ Mediterranean Tropical; Med. Atl. = Mediterranean Atlantic; Euro-Med. = Euro-Mediterranean; Euro-Med.-Iran.-Tur = Euro-Mediterranean-IranoTuranian; Med.-Iran.-Tur. $=$ Mediterranean-Irano-Turanian; Paleotemp. $=$ Paleotemperate; Circumbor = Circumboreal; Cosmop. $=$ Cosmopolitan (chorological types adapted from Brullo et al., 1998).

\begin{tabular}{|c|c|c|c|c|c|}
\hline Family & Species & Life Form & $\begin{array}{l}\text { Chorological } \\
\text { type }\end{array}$ & Coll. & Notes \\
\hline Poaceae & Aeluropus lagopoides (L.) Trin. & G rhiz & Med.-Trop. & Ac & \\
\hline Alliaceae & Allium commutatum Guss. & G bulb & Med. & Ac & NV \\
\hline Apiaceae & Ammi majus L. & T scap & Med. & Ac & nr, NV \\
\hline Poaceae & $\begin{array}{l}\text { Ammophila australis (Mabille) Porta \& } \\
\text { Rigo }\end{array}$ & H caesp & Med. & Ac & \\
\hline Poaceae & Anisantha rigida (Roth) Hyl. & T scap & Med. & Ac & \\
\hline Asteraceae & Anthemis secundiramea Biv. & T scap & C Med. & Ac & \\
\hline Chenopodiaceae & $\begin{array}{l}\text { Arthrocnemum macrostachyum (Moric.) } \\
\text { Moris }\end{array}$ & Ch succ & Med.-Iran.-Tur. & Ac & \\
\hline Asparagaceae & Asparagus acutifolius L. & NP & Med. & Ac & \\
\hline Asphodelaceae & Asphodelus ramosus L. subsp. ramosus & G rhiz & Med. & Ac & \\
\hline Asteraceae & Asteriscus aquaticus (L.) Less. & G bulb & Med. & Ac & \\
\hline Fabaceae & Astragalus boeticus L. & T scap & Med.-Iran.-Tur. & $\mathrm{n}$ & \\
\hline Poaceae & Avena fatua L. & T scap & Cosmop. & Ac & nr, NV \\
\hline Chenopodiaceae & Beta maritima L. & H scap & Paleotemp. & Ac & \\
\hline Brassicaceae & Cakile maritima Scop. & T scap & Med. & Ac & \\
\hline Poaceae & $\begin{array}{l}\text { Catapodium balearicum (Willk.) H. } \\
\text { Scholz }\end{array}$ & T scap & Med. & Ac & \\
\hline Poaceae & $\begin{array}{l}\text { Catapodium rigidum (L.) Hubbard } \\
\text { subsp. rigidum }\end{array}$ & T scap & $\begin{array}{l}\text { Euro-Med.- } \\
\text { Iran.-Tur }\end{array}$ & Ac & \\
\hline Asteraceae & Centaurea sicula L. & H bien & O Med. & Ac & \\
\hline Asteraceae & Centaurea sphaerocephala L. & H scap & Med. & Ac & \\
\hline Hyacinthaceae & Charybdis pancration (Steinh.) Speta & G bulb & Med. & Ac & \\
\hline Convolvulaceae & Convolvulus elegantissimus Miller & H scand & O Med. & Ac & \\
\hline Apiaceae & Crithmum maritimum L. & Ch suffr & Med.-Atl. & Ac & \\
\hline Poaceae & Cutandia divaricata (Desf.) Bentham & T scap & O-Med. & $\mathrm{n}$ & \\
\hline Apiaceae & Daucus carota L. subsp. carota & H bien & Euro-Med. & Ac & \\
\hline Asteraceae & Dittrichia viscosa (L.) Greuter & H scap & O Med. & Ac & \\
\hline Apiaceae & Echinophora spinosa L. & H scap & Med. & $\mathrm{n}$ & \\
\hline Boraginaceae & Echium sabulicola Pomel & H scap & O Med. & $\mathrm{n}$ & \\
\hline Poaceae & Elytrigia juncea (L.) Nevski & G Rhiz & Med. & $\mathrm{n}$ & \\
\hline
\end{tabular}




\begin{tabular}{|c|c|c|c|c|c|}
\hline Poaceae & Elytrigia repens (L.) Desv. & G rhiz & Paleotemp. & Ac & nr, pm \\
\hline Ephedraceae & Ephedra fragilis Desf. & NP caesp & Med. & Ac & \\
\hline Geraniaceae & Erodium chium (L.) Willd. & T scap & Med. & Ac & nr NV \\
\hline Geraniaceae & Erodium laciniatum (Cav.) Willd. & T scap & Med. & $\mathrm{n}$ & \\
\hline Geraniaceae & Erodium malacoides (L.) L'Her. & T scap & Med. & Ac & \\
\hline Apiaceae & Eryngium maritimum L. & G rhiz & Med.-Atl. & Ac & \\
\hline Euphorbiaceae & Euphorbia paralias L. & Ch frut & Med.-Atl. & Ac & \\
\hline Euphorbiaceae & Euphorbia pinea L. & Ch suffr & Med. & Ac & \\
\hline Euphorbiaceae & Euphorbia terracina L. & T scap & Med. & Ac & \\
\hline Apiaceae & Foeniculum vulgare L. subsp. vulgare & H scap & S Med. & Ac & \\
\hline Frankeniaceae & Frankenia hirsuta L. & Ch suffr & Med.-Iran.-Tur. & Ac & \\
\hline Asteraceae & Galactites elegans (All.) Soldano & H bien & Med. & $\mathrm{n}$ & \\
\hline Papaveraceae & Glaucium flavum Crantz & H scap & Circumbor. & $\mathrm{n}$ & \\
\hline Asteraceae & Glebionis coronaria (L.) Spach & T scap & Med. & Ac & \\
\hline Chenopodiaceae & Halimione portulacoides (L.) Aellen & Ch frut & Circumbor. & $\mathrm{n}$ & \\
\hline Poaceae & Hordeum murinum $\mathrm{L}$. & T scap & Circumbor. & Ac & nr, pm \\
\hline Poaceae & Hordeum leporinum Link & T scap & Euro-Med. & $\mathrm{n}$ & \\
\hline Juncaceae & Juncus capitatus Weigel & T scap & Med.-Atl. & Ac & \\
\hline Poaceae & Lagurus ovatus L. subsp. ovatus & T scap & Med. & Ac & \\
\hline Asteraceae & Launaea fragilis (Asso) Pau & Ch frut & S Med. & $\mathrm{n}$ & \\
\hline Malvaceae & Lavatera arborea $\mathrm{L}$. & $\mathrm{H}$ bien & Med. & $\mathrm{n}$ & \\
\hline Malvaceae & Lavatera cretica L. & T scap & Med. & Ac & $\mathrm{nr}, \mathrm{pm}$ \\
\hline Asteraceae & $\begin{array}{l}\text { Limbarda crithmoides (L.) Dumort. } \\
\text { subsp. longifolia (Arcang.) Greuter }\end{array}$ & Ch suffr & Med. & Ac & \\
\hline Plumbaginaceae & Limonium narbonense Miller & $\mathrm{H}$ ros & Med. & Ac & \\
\hline Plumbaginaceae & Limonium syracusanum Brullo & Ch suffr & Hyblean End. & $\mathrm{n}$ & \\
\hline Fabaceae & Lotus creticus L. & Ch suffr & Med. & Ac & \\
\hline Solanaceae & Mandragora autumnalis Bertol. & $\mathrm{H}$ ros & Med. & Ac & \\
\hline Brassicaceae & Matthiola tricuspidata (L.) R. Br. & T scap & Med. & Ac & \\
\hline Fabaceae & Medicago ciliaris (L.) All. & T scap & Med. & Ac & \\
\hline Euphorbiaceae & Mercurialis anпua $\mathrm{L}$. & T scap & Paleotemp. & Ac & \\
\hline Aizoaceae & Mesembryanthemum nodiflorum $\mathrm{L}$. & T scap & Med.-Trop. & Ac & \\
\hline Cactaceae & Opuntia ficus-indica (L.) Miller & P succ & Nat. (Neotrop.) & $\mathrm{n}$ & \\
\hline Amaryllidaceae & Pancratium maritimum L. & G bulb & Med. & Ac & \\
\hline Poaceae & $\begin{array}{l}\text { Parapholis filiformis (Roth) C. E. } \\
\text { Hubbard }\end{array}$ & T scap & O Med. & Ac & \\
\hline Poaceae & Parapholis incurva (L.) Hubbard & T scap & Med.-Atl. & Ac & \\
\hline Poaceae & Phalaris canariensis $\mathrm{L}$. & T scap & Euro-Med. & Ac & \\
\hline Poaceae & $\begin{array}{l}\text { Piptatherum miliaceum (L.) Coss. subsp. } \\
\text { miliaceum }\end{array}$ & H caesp & Med.-Atl. & Ac & \\
\hline Apiaceae & Pseudorlaya pumila (L.) Grande & T scap & Med. & Ac & \\
\hline
\end{tabular}




\begin{tabular}{|c|c|c|c|c|c|}
\hline Iridaceae & Romulea columnae Seb. \& Mauri & G bulb & Med. & Ac & \\
\hline Polygonaceae & $\begin{array}{l}\text { Rumex bucephalophorus L. subsp. } \\
\text { bucephalophorus }\end{array}$ & T scap & Med. & Ac & \\
\hline Chenopodiaceae & Kali turgidum (Dumort.) Gutermann. & T scap & Euro-Atl. & Ac & $\mathrm{nr}, \mathrm{pm}$ \\
\hline Chenopodiaceae & Kali tragus (L.) Scop. & T scap & Paleotemp. & Ac & \\
\hline Chenopodiaceae & Sarcocornia fruticosa (L.) A.J. Scott & Ch succ & Boreo-Trop. & Ac & \\
\hline Rosaceae & Sarcopoterium spinosum (L.) Spach & NP & E Med. & Ac & \\
\hline Asteraceae & Scolymus hispanicus L. & H bien & Med. & $\mathrm{n}$ & \\
\hline Crassulaceae & Sedum rubens $\mathrm{L}$. & T scap & Med.-Atl. & Ac & \\
\hline Asteraceae & $\begin{array}{l}\text { Senecio glaucus L. subsp. coronopifolius } \\
\text { (Maire) Alex. }\end{array}$ & T scap & Med.-Iran.-Tur. & Ac & NV \\
\hline Asteraceae & Senecio leucanthemifolius Poiret & T scap & O Med. & $\mathrm{n}$ & NV \\
\hline Apiaceae & $\begin{array}{l}\text { Seseli tortuosum L. subsp. maritimum } \\
\text { Brullo et al. }\end{array}$ & H bien & $\begin{array}{l}\text { Sicily, Sardinia, } \\
\text { Calabria End. }\end{array}$ & $\mathrm{n}$ & \\
\hline Lamiaceae & Sideritis romana $\mathrm{L}$. & T scap & O Med. & Ac & \\
\hline Caryophyllaceae & Silene colorata Poiret & T scap & Med. & $\mathrm{n}$ & \\
\hline Caryophyllaceae & Silene nicaeensis All. & T scap & Med. & Ac & \\
\hline Caryophyllaceae & Silene sedoides Poiret & T scap & Med. & Ac & \\
\hline Caryophyllaceae & $\begin{array}{l}\text { Silene vulgaris (Moench) Garcke subsp. } \\
\text { tenoreana (Colla) Soldano \& F. Conti }\end{array}$ & H scap & Med. & Ac & \\
\hline Asteraceae & Sonchus oleraceus L. & T scap & Cosmop. & $\mathrm{n}$ & \\
\hline Asteraceae & Sonchus tenerrimus L. & H scap & Med. & Ac & $\mathrm{nr}$ \\
\hline Caryophyllaceae & Spergularia salina J. \& C. Presl & T scap & Paleotemp. & $\mathrm{n}$ & \\
\hline Poaceae & Stipa capensis Thumb. & T scap & Med. & Ac & \\
\hline Chenopodiaceae & Suaeda vera J. F. Gmelin & NP caesp & Med.-Atl. & Ac & \\
\hline Fabaceae & Vicia villosa Roth subsp. villosa & T scap & Euro-Med. & $\mathrm{n}$ & \\
\hline Poaceae & Vulpia fasciculata (Forssk) Fritsch & Tscap & Med. & $\mathrm{n}$ & \\
\hline
\end{tabular}

are listed according to DeviLlers (1991), EUNIS habitat codes follow DAvies et al. (2004) and the habitats of European Directive 92/43 CE are listed according to EuropeAn ComMission, (2013) and Biondi \& BLASI (2010).

\section{RESULTS}

\section{Flora}

According to the list published by Albo (1959), who recorded 67 taxa, and our collections and observation, the vascular flora of Vendicari islet consists of 88 specific and infraspecific taxa (Tab. 1). Excluding the 8 species we did not find ( $\mathrm{nr}$ in the list), some of which were probably misidentified by Aцво (1959), the actual flora now consists of 80 taxa.

The life form spectrum of actual flora indicates the predominance of therophytes $(40$ species, $48 \%)$, with a relevant presence hemicryptophytes $(20,24 \%)$, and geophytes (8, 
Tab. 2. Vendicari Islet plant communities.

\begin{tabular}{|c|c|c|c|c|c|c|c|c|c|c|c|c|c|c|c|c|c|c|c|}
\hline Releve Nr. & 1 & 2 & 3 & 4 & 5 & 6 & 7 & 8 & 9 & 10 & 11 & 12 & 13 & 14 & 15 & 16 & 17 & 18 & 19 \\
\hline Area (m2) & 5 & 10 & 10 & 50 & 50 & 40 & 50 & 50 & 40 & 40 & 50 & 5 & 5 & 2 & 50 & 90 & 100 & 40 & 1 \\
\hline Cove & 60 & 90 & 70 & 90 & 90 & 90 & 85 & 85 & 85 & 90 & 85 & 70 & 90 & \begin{tabular}{l|l}
90 & 1
\end{tabular} & 100 & 90 & 50 & 80 & 80 \\
\hline Substrate & $\mathrm{s}$ & $\mathrm{s}$ & $\mathrm{s}$ & $\mathrm{s}$ & $\mathrm{s}$ & c & $\mathrm{s}$ & $\mathrm{s}$ & $\mathrm{s}$ & $\mathrm{s}$ & s & S & S & $\mathrm{s}$ & c & c & c & c & $\mathrm{s}$ \\
\hline Corine Biotopes codes & 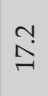 & $\begin{array}{l}\mathbb{N} \\
\stackrel{N}{\rightarrow}\end{array}$ & $\begin{array}{l}\text { No } \\
\stackrel{5}{N}\end{array}$ & $\begin{array}{l}\mathbb{N} \\
\stackrel{N}{n}\end{array}$ & $\begin{array}{l}\stackrel{-}{N} \\
\stackrel{5}{2}\end{array}$ & $\begin{array}{c}-\overrightarrow{0} \\
\stackrel{1}{2} \\
-\end{array}$ & $\begin{array}{l}\vec{\sim} \\
\text { ğ }\end{array}$ & $\begin{array}{l}\text { הิ } \\
\text { ڤ్- }\end{array}$ & 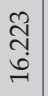 & 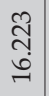 & $\begin{array}{l}\text { స్ } \\
\text { } \\
-\end{array}$ & 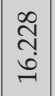 & 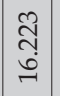 & $\begin{array}{l}\underset{ే}{ } \\
\text { } \\
-1\end{array}$ & $\begin{array}{l}\vec{\infty} \\
\dot{+} \\
\end{array}$ & $\begin{array}{l}\vec{\infty} \\
\dot{\infty} \\
\dot{\oplus}\end{array}$ & $\begin{array}{l}-\overrightarrow{0} \\
\stackrel{1}{2} \\
\sim\end{array}$ & $\begin{array}{l}\vec{b} \\
\stackrel{1}{\sim}\end{array}$ & 군 \\
\hline Habitat 92/43 EEC codes & $\stackrel{ }{\mathfrak{I}}$ & สิ & สิ & ฐิ & กิ & I & $\stackrel{ }{\vec{N}}$ & 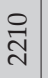 & $\stackrel{ }{\grave{2}}$ & $\stackrel{ }{\stackrel{\Xi}{\Xi}}$ & 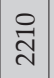 & 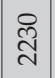 & สิ & ๙্ণ & 1 & 1 & 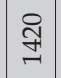 & ฐิ & $\frac{0}{m}$ \\
\hline \multicolumn{20}{|c|}{ CAKILION MARITIMAE \& CAKILETEA MARITIMAE } \\
\hline Matthiola tricuspidata & 3 & + & . & + & + & . & + & + & & . & & . & & . & . & . & & . & . \\
\hline Glaucium flavum & + & $\cdot$ & . & . & $\cdot$ & . & . & . & & . & . & . & & . & . & . & 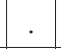 & . & . \\
\hline Cakile maritima & + &. & . & . & & . & + & & & 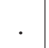 & + & . & & 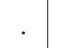 & & 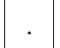 & & . & \\
\hline \multicolumn{20}{|c|}{ CRUCIANELLETALIA MARITIMAE \& EUPHORBIO-AMMOPHILETEA AUSTRALIS } \\
\hline Elytrigia juncea & . & 1 & + & + & + & . & 4 & 1 & + & 1 & + & . & . & . & . & . & . & . & . \\
\hline Centaurea sphaerocephala & & . & . & . & & . & 1 & 4 & + & + & 5 & . & & & & . & . & . & . \\
\hline $\begin{array}{l}\text { Seseli tortuosum subsp. } \\
\text { maritimum }\end{array}$ & . & 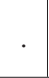 & $\cdot$ & . & $\cdot$ & . & 2 & 1 & 3 & 4 & 2 & + & + & + & . & . & . & . & . \\
\hline Euphorbia terracina & . & $\cdot$ & . & + & + & . & + & + & 1 & + & + & . & . & . & . & . & . & . & . \\
\hline Lotus creticus & + &. & . & + & . & . & 1 & 2 & 2 & 3 & 1 & + & + & + & + & & . & . & . \\
\hline Pancratium maritimum & . & $\cdot$ & . &. & . & . & + & + & 1 & + & 1 & . & . & . & . & . & . & . & . \\
\hline Launaea fragilis & . &. & . & . & . & . & + & + & 2 & 1 & + & . & . & . & . & & . & . & $0^{\circ}$ \\
\hline Echinophora spinosa & . & & . & & . & & + & + & $\cdot$ & . & . & . & . & & . & & . & . & . \\
\hline Scolymus hispanicus & . & $\cdot$ & . & & & . & . & . & . & . & + & . & . & . & . & . & . & . & . \\
\hline \multicolumn{20}{|c|}{ SARCOCORNIETEA FRUTICOSAE } \\
\hline Suaeda vera & . & 4 & 2 & 4 & 5 & 5 & . & . & . & . & . & . & . & . & 1 & 2 & 2 & . & . \\
\hline $\begin{array}{l}\text { Arthrocnemum mac- } \\
\text { rostachyum }\end{array}$ & . & r. & $\cdot$ & . & . & 1 & . & . & & . & . & . & & . & . & . & 3 & 4 & . \\
\hline Halimione portulacoides & . &. & 3 & . & . & + & . & . & 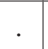 & . & . & . & & . & . & . & . & . & . \\
\hline Sarcocornia fruticosa & & . & . & . & . & + & . & . & & . & & . & & . & & . & . & . & . \\
\hline Limonium narbonense & . & $\cdot$ & . & + & 1 & 1 & . & . & . & . & + & . & . & . & . & . & + & + & . \\
\hline \multicolumn{20}{|c|}{ CRITHMO MARITIMI-LIMONIETEA } \\
\hline Crithmum maritimum & . & 2 & + & 1 & 1 & . & . & + & + & . & . & . & . & . & . & . & + & . & . \\
\hline Frankenia hirsuta & . & $\cdot$ & . & $\cdot$ & . & . & . & . & . & . & . & . & . & . & . & . & + & + & 1 \\
\hline Limonium syracusanum & $\cdot$ & $\cdot$ & . &. & . & . & . & . & . & . & . & . & . & . & . & . & 1 & . & . \\
\hline \multicolumn{20}{|c|}{ CUTANDIETALIA MARITIMAE \& HELIANTHEMETEA GUTTATI } \\
\hline Cutandia divaricata & $\cdot$ & $\cdot$ & . & $\cdot$ & . & . & + & + & . & . & . & 4 & 3 & 3 & . & . & . & . & . \\
\hline $\begin{array}{l}\text { Lagurus ovatus subsp. } \\
\text { ovatus }\end{array}$ & $\cdot$ & $\cdot$ & $\cdot$ & . & r. & $\cdot$ & + & + & . & . & . & + & + & 1 & . & & . & . & \\
\hline
\end{tabular}




\begin{tabular}{|c|c|c|c|c|c|c|c|c|c|c|c|c|c|c|c|c|c|c|c|}
\hline Silene colorata & . & . & . & . & . & $\cdot$ & . & . & . & . & . & 1 & 2 & 1 & . & - & . & $\cdot$ & . \\
\hline Vulpia fasciculata & . & . & $\cdot$ & . & $\cdot$ & . & + & + & . & . & . & 2 & 3 & 2 & $\cdot$ & . & . & . & . \\
\hline Senecio leucanthemifolius & . & . & . & . & . & $\cdot$ & $\cdot$ & . & . & . & . & + & + & + & . & . & . & . & . \\
\hline Erodium laciniatum & . & . & . & $\cdot$ & . & . & + & . & . & . & . & + & + & + & . & $\cdot$ & . & . & . \\
\hline Echium sabulicolum & . & . & . & . & . & . & . & . & . & . & . & + & + & . & $\cdot$ & $\cdot$ & . & . & . \\
\hline Senecio coronopifolius & . & $\cdot$ & . & . & . & $\cdot$ & + & + & . & . & $\cdot$ & $\cdot$ & $\cdot$ & . & $\cdot$ & . & . & $\cdot$ & . \\
\hline \multicolumn{20}{|c|}{ FRANKENION PULVERULENTAE E SAGINETEA MARITIMAE } \\
\hline Spergularia salina & . & . & . & . & . & . & . & . & . & . & . & . & . & . & . & . & . & . & 3 \\
\hline Parapholis filiformis & . & . & . & . & . & . & $\cdot$ & . & . & . & . & . & . & . & . & . & . & . & 2 \\
\hline Parapholis incurva & . & . & . & $\cdot$ & . & . & . & . & . & $\cdot$ & . & . & . & . & . & . & . & + & 1 \\
\hline \multicolumn{20}{|c|}{ THERO-BROMETALIA \& STELLARIETEA MEDIAE } \\
\hline Mercurialis annua & . & . & . & $\cdot$ & . & . & . & . & . & . & . & . & . & . & 2 & 1 & . & . & . \\
\hline Sonchus oleraceus & 1 & + & + & + & + & . & + & + & . & $\cdot$ & . & + & . & + & 2 & 1 & . & . & . \\
\hline Anisantha rigida & . & . & . & . & . & . & . & + & . & . & + & + & + & 1 & 2 & + & . & . & . \\
\hline Astragalus boeticus & . & . & . & . & . & $\cdot$ & . & . & . & $\cdot$ & . & . & . & . & 1 & . & . & . & . \\
\hline Vicia villosa subsp. villosa & . & . & . & . & . & . & . & . & . & . & . & . & . & . & + & . & . & . & . \\
\hline Erodium malacoides & . & . & . & . & . & . & . & . & . & $\cdot$ & $\cdot$ & . & . & . & 1 & . & . & & . \\
\hline Dittrichia viscosa & . & . & . & $\cdot$ & . & . & . & . & . & . & . & . & . & . & + & 1 & . & . & . \\
\hline Glebionis coronaria & . & . & . & . & . & . & & . & . & $\cdot$ & + & + & + & . & 1 & + & . & . & . \\
\hline Galactites elegans & . & . & . & . & . & . & . & . & . & . & . & . & . & . & 1 & $\cdot$ & . & . & . \\
\hline Lavatera arborea & . & . & . & . & . & . & . & . & . & $\cdot$ & $\cdot$ & . & . & . & . & 3 & . & . & . \\
\hline \multicolumn{20}{|l|}{ Other species } \\
\hline Asparagus acutifolius & . & . & . & . & . & . & . & + & + & + & + & . & . & . & . & . & . & . & . \\
\hline Ephedra fragilis & . & . & . & . & . & . & + & . & + & + & + & . & . & . & . & . & . & . & . \\
\hline $\begin{array}{l}\text { Convolvulus } \\
\text { elegantissimus }\end{array}$ & . & . & . & . & . & . & . & . & . & . & . & . & . & . & 2 & 2 & . & . & . \\
\hline Opuntia ficus-indica & . & . & . & . & . & . & $\cdot$ & . & . & . & $\cdot$ & . & . & . & + & + & . & . & . \\
\hline Oryzopsis miliacea & . & . & . & . & . & . & . & . & . & . & . & . & . & . & 1 & 1 & . & . & . \\
\hline Asphodelus ramosus & $\cdot$ & . & . & . & . & . & . & . & . & . & . & . & . & . & 1 & . & . & . & . \\
\hline Charybdis pancration & . & . & . & . & . & . & . & . & + & . & . & . & . & . & + & . & . & . & . \\
\hline Beta maritima & + & . & . & . & . & . & . & . & . & . & . & . & . & . & . & . & . & . & . \\
\hline Euphorbia pinea & . & . & . & + & . & . & . & . & . & . & . & . & . & . & . & . & . & . & . \\
\hline
\end{tabular}

Rels. 1-19: Isola di Vendicari, 10th May 2012. Substrate: $\mathrm{s}=$ sandy, c = calcarenitic. Rel. 1: Glaucio flavi-Matthioletum tricuspidatae; Rels. 2-6: Halimiono portulacoidis-Suaedetum verae; Rel. 7: Cypero mucronati-Elytrigietum junceae; Rels. 8-11: Centaureo-Ononidetum ramosissimae; Rels. 12-14: Vulpio-Cutandietum divaricatae; Rels. 15-16: Thero-Brometalia; Rels. 1718: Limonio virgati-Arthrocnemetum macrostachyi; Rel. 19: Parapholidetum filiformis.

9\%) (Fig. 2). The unique phanerophytes are Ephedra fragilis, a maquis species, and a few specimens of Opuntia ficus indica introduced by man during last century.

From the chorological viewpoint (Fig. 3) the greatest number of plants belong to the Mediterranean chorological element (54 species, 66\%), including the Hyblaean endemic 


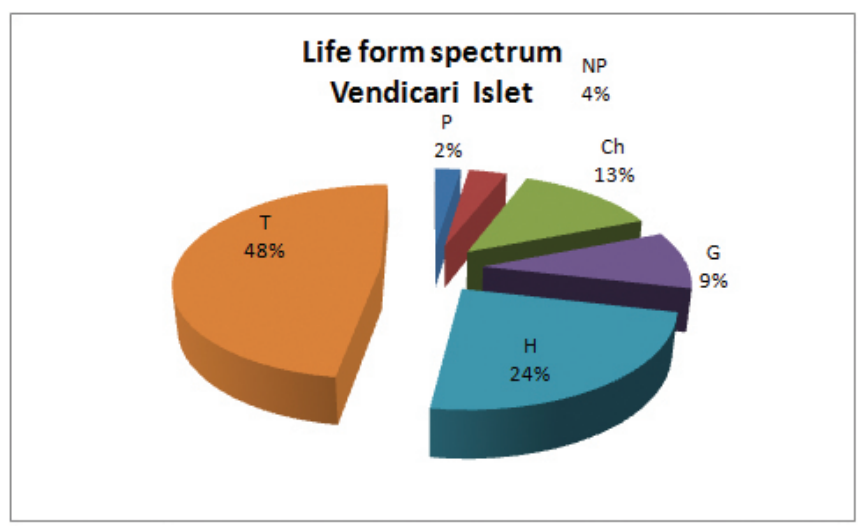

Fig. 2. Life-form spectrum of Vendicari Islet.

Limonium syracusanum, (BRULlo et al., 2011a), Seseli tortuosum subsp. maritimum endemic of Sicily, Sardinia and west Calabria (Brullo et al., 2011b); dominant inside the group is the Circum-Mediterranean element (40 species, $48 \%$ ) and there is a relevant presence of W Mediterranean species (8, 10\%), (Fig. 3). Among species with distribution areas extending outside of the Mediterranean area the most significant are the Atlantic-Mediterranean ones $(8,10 \%)$.

\section{Plant communities and habitats}

The islet is very low (no more than $2.5 \mathrm{~m}$ a.s.1.), so it is completely exposed to salt and sea aerosol influences, but the edaphic conditions show different features. Therefore, the vegetation is strictly correlated to the ecological factors, which have a decisive effect on the distribution of the plant communities on the islet. The recognized vegetation types are shown in the Tab. 2 according to the phytosociological method, mainly based on the

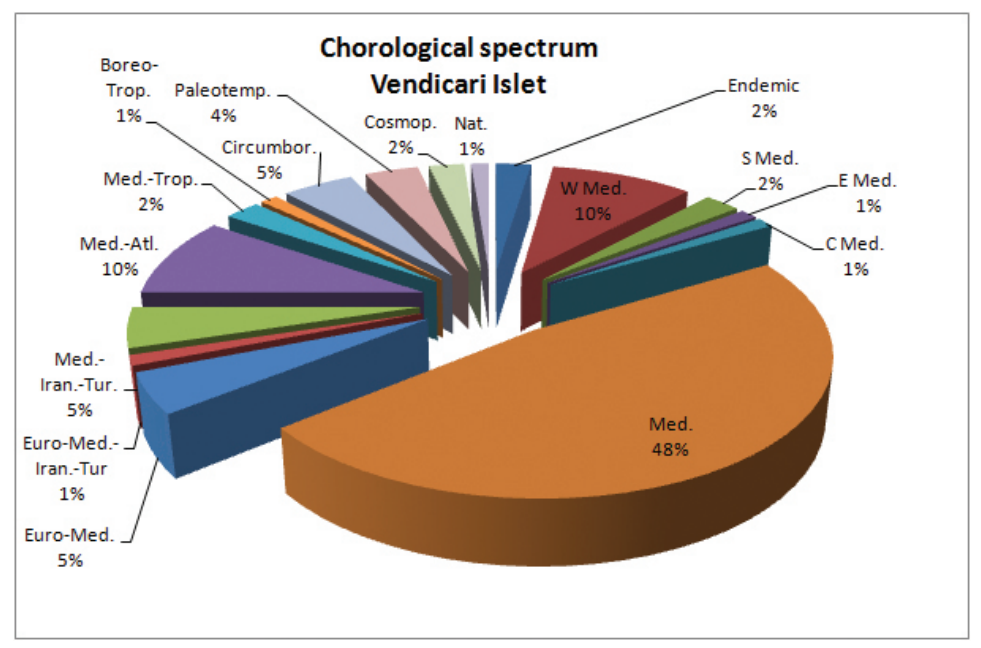

Fig. 3. Chorological spectrum of Vendicari Islet. 


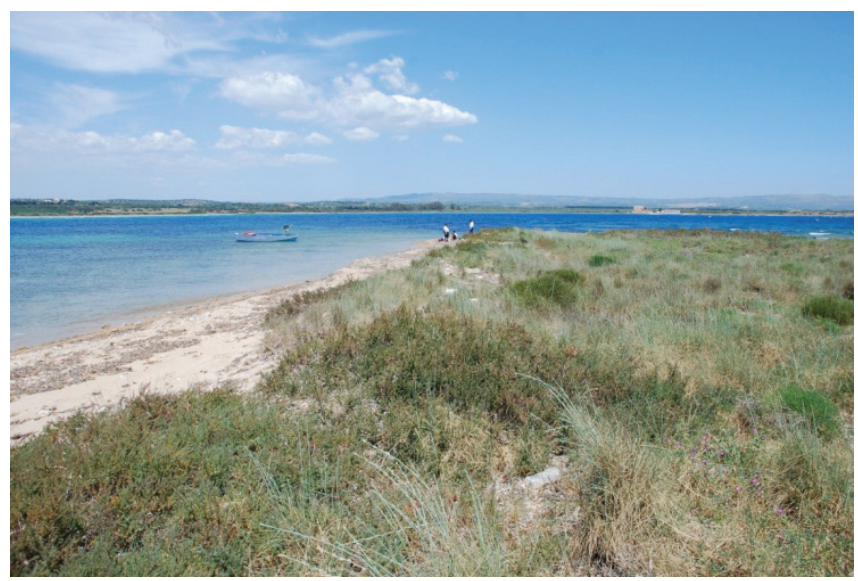

Fig. 4. The east side of the islet with Elytrigia juncea community and some Suaeda vera shrub (photo by: P. Minissale)

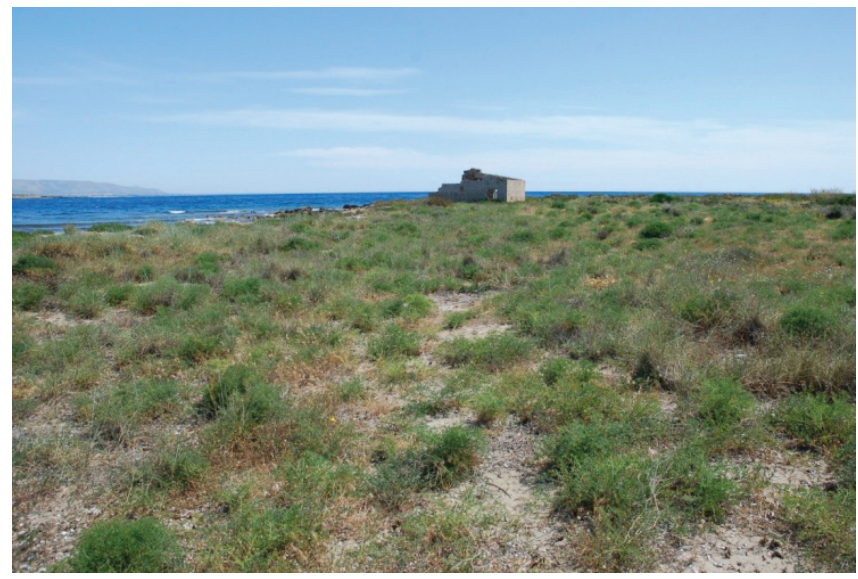

Fig. 5. Plant community with Seseli tortuosum subsp. maritimum and Centaurea sphaerocephala (photo by: P. Minissale)

previous investigations carried out in Vendicari area and more generally of the south eastern coastal Sicily by Brullo et al. (1980), BARTolo et al. (1982) and Minissale \& SCIANDRELLO (2010). In particular, the low sandy belt near the sea is colonized by annual halo-nitrophilous vegetation belonging to Cakiletea maritimae R.Tx. \& Preising in Br.-Bl. \& R.Tx.1952, where Cakile maritima, Matthiola tricuspidata, Glaucium flavum have their optimum growth. This community can be attributed to Glaucio flavi-Matthioletum tricuspidatae Blasi, Fascetti, Veri \& Bruno 1983 (Rel. 1), previously quoted from Sicily by Marcenò \& Romano (2010). Some embryonic dunes are colonized by an impoverished vegetation characterized by Elytrigia juncea referable to Cypero mucronati-Elytrigietum junceae Kühnholtz-Lordat ex Br.-Bl. 1933 (Rel. 7), association of the class Ammophiletea Br.-Bl. \& Tüxen ex Westhoff, Dijk \& Passchier 1946 (Fig. 4). On organic accumulations near the sea, consisting mainly of beached fragments of Posidonia oceanica, there is a 


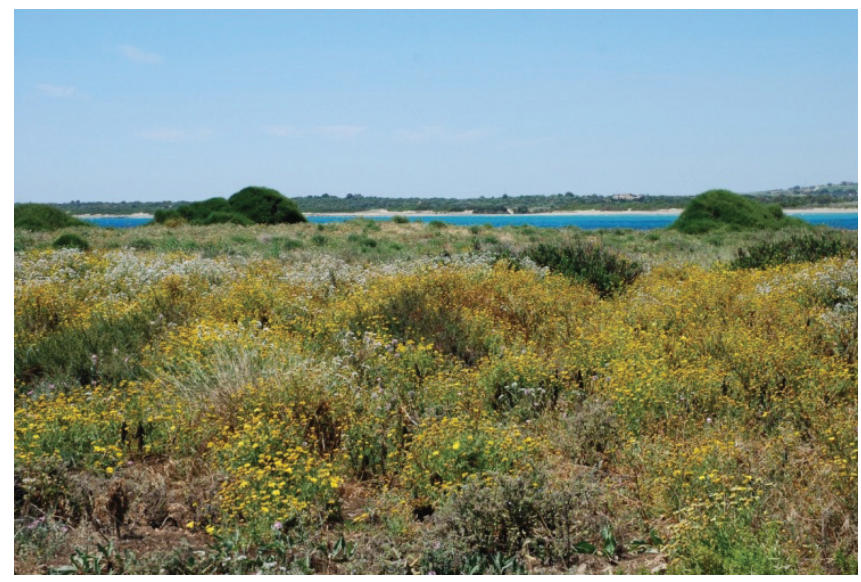

Fig. 6. Nitrophilous vegetation with Glebionis coronaria; Ephedra fragilis and the dunes of Vendicari coast in the background (photo by: P. Minissale)

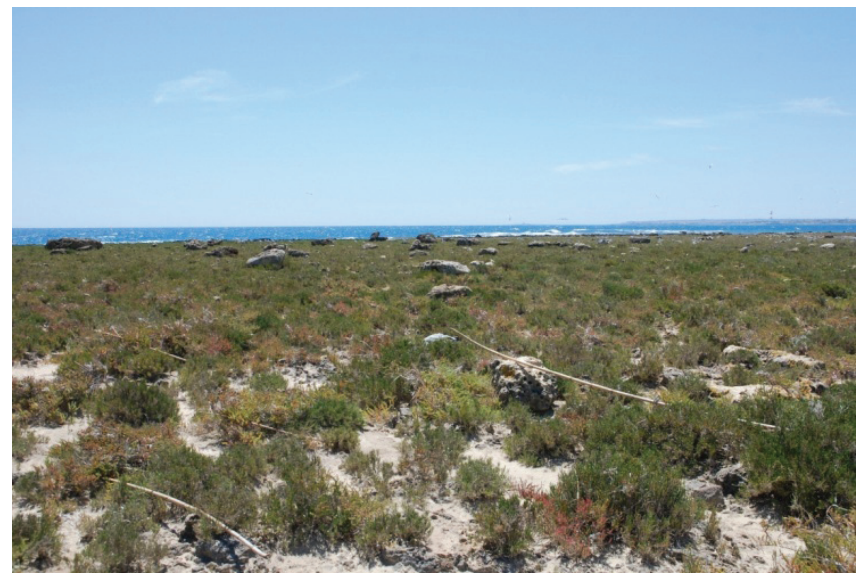

Fig. 7. The south side of the islet with the Arthrocnemum macrostachyum community where Audouin's Gull nests (photo by: P. Minissale)

perennial plant community dominated by Suaeda vera associated with other halophilous species, such as Halimione portulacoides and Crithmum maritimum. This halo-subnitrophilous vegetation is referable to Halimiono portulacoidis-Suaedetum verae Molinier \& Tallon 1970 (Rels. 2-6), an association belonging to Suaedion verae Brullo \& Furnari 1988, alliance of Sarcocornietea fruticosae Br.-Bl. \& R.Tx. ex A. \& O.Bolòs 1950. The central part of islet, with stabilized sandy soil, is colonized by psammophilous vegetation with species of Crucianelletalia maritimae Sissing 1974, such as Sesesli tortuosum subsp. maritimum, Centaurea sphaerocephala, Lotus creticus, Euphorbia terracina, Launaea fragilis, etc. (Fig. 5). On the islet Ononis hispanica subsp. ramosissima (Desf.) Förther \& Podlech is lacking, although it is very common in the coast in front of the islet, so this community represents a simplified aspect of Centaureo-Ononidetum ramosissimae Br.-Bl. \& Frei in Frei 1937 (Rels. 8-11). Within this vegetation, in the small clearings, there are frequent ephemeral dry 


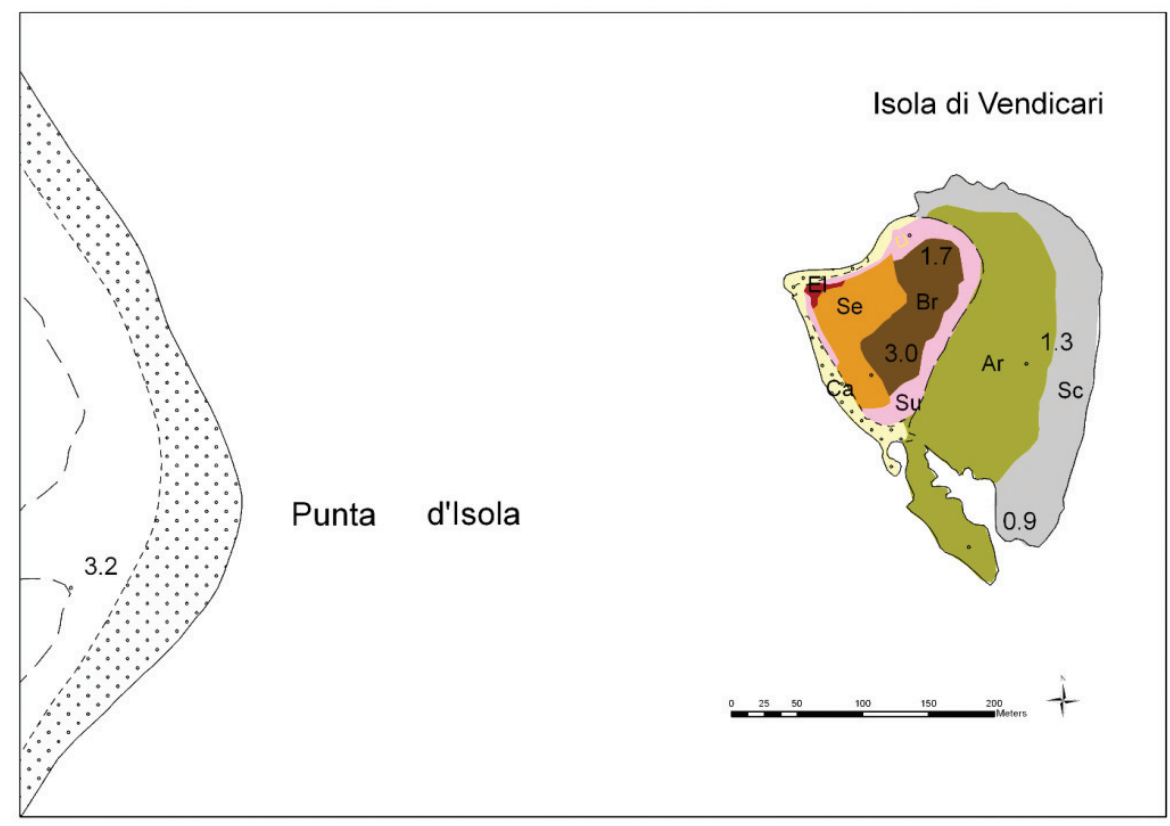

Fig. 8. Vegetation map: Ca - Glaucio flavi-Matthioletum tricuspidatae; $\mathrm{Su}$ - Halimiono portulacoidis-Suaedetum verae; E1 - Cypero mucronati-Elytrigietum junceae; Se - Centaureo-Ononidetum ramosissimae; $\mathrm{Br}$ - Thero-Brometalia; $\mathrm{Ar}$ - Limonio virgati-Arthrocnemetum glauci; $\mathrm{Sc}$ - Reef

grasslands of Cutandietalia divaricatae Rivas-Martìnez, Diez-Garretas \& Asensi 2002, characterized by psammophilous therophytes represented mainly by Cutandia divaricata and Vulpia fasciculata, which can be attributed to Vulpio-Cutandietum divaricatae Brullo \& Scelsi 1998 (Rels. 12-14), an association distributed in southern Sicily. The calcarenitic rocks occurring along the eastern side of the islet are more exposed to the sea storms and aerosol, but are covered in the inner part by a community dominated by Arthrocne-

Tab. 3. Surfaces and European codes (Corine Biotopes, EUNIS and Habitat of Annex I Dir. 92/43) of the habitats occurring in the islet.

\begin{tabular}{|c|c|c|c|c|}
\hline $\begin{array}{c}\text { CORINE } \\
\text { Biotopes }\end{array}$ & EUNIS & $\begin{array}{c}\text { Habitat 92/43 } \\
\text { CE }\end{array}$ & $\begin{array}{c}\text { Acronym used in Fig. 8 } \\
\text { (Vegetation map) }\end{array}$ & $\begin{array}{c}\text { Surface } \\
\text { (hectares) }\end{array}$ \\
\hline 15.61 & A2.5 & 1420 & Ac & 1.780 \\
\hline 15.61 & A2.5 & 1420 & Su & 0.373 \\
\hline 16.211 & B1.3 & 2110 & E1 & 0.022 \\
\hline 16.223 & B1.4 & 2210 & Se & 0.408 \\
\hline 17.2 & B1.12 & 1210 & Ca & 0.265 \\
\hline 18.16 & B3.1 & 0 & Sc & 1.140 \\
\hline 34.81 & E1.6 & 0 & Br & 0.462 \\
\hline & & Total & & 4.450 \\
\hline
\end{tabular}


mum macrostachyum, which is linked to salt soils (Fig. 7). This vegetation in terms of its ecology and physiognomic aspect is very similar to Limonio virgati-Arthrocnemetum macrostachyi Biondi, Casavecchia \& Guerra 2006 (Rels. 17-18), an association of Sarcocornietea fruticosae, described by Biond et al. (2006), for Apulia on low calcarenitc cliffs. Frequently mixed with this vegetation is an ephemeral community dominated by halonitrophylous microphytes of Saginetea maritimae Westhoff, Van Leeuwen \& Adriani 1962, such as Parapholis filiformis, P. incurva and Spergularia salina. This vegetation is referable to Parapholidetum filiformis Brullo, Scelsi \& Siracusa 1994 (Rel. 19), an association widespread along the Sicilian coasts.

In the central part of the islet on soils enriched with nitrates, a legacy of human activities, now very distant in time, there is nitrophilous vegetation. It is a very heterogeneous community rich in annual therophytes mixed to some perennial species which can be attributed to Thero-Brometalia (Rivas Goday \& Rivas-Martínez ex Esteve 1973) O.Bolòs 1975 order of Stellarietea mediae R.Tx., Lohmeyer \& Preising ex von Rochow 1951 (Fig. 6).

The surfaces of these different habitats with their European codes (Corine Biotopes, EUNIS and Habitat of Annex I Dir. 92/43), are reported in Tab. 3. They are also mapped, as vegetation types, in Fig. 8.

\section{DISCUSSION}

The floristic settlement of the islet shows close relations with the flora of the not very distant Sicilian coast, so it could be interesting to compare the Vendicari Islet floristic list to the flora of Vendicari Nature Reserve. In particular according to Minissale \& ScianDRELLO (2010), the flora of this reserve (the islet excluded) consists of 486 taxa and almost all the species occurring on the islet are shared with this flora. The islet species not recorded for the Vendicari reserve are trivial and probably previously escaped observation or were misidentified.

As regard the colonization processes, it can be clearly hypothesized that the species with good dispersion ability reached the islet by the wind, sea currents or zoochoria. Moreover it has to be taken into account the proximity to the Sicilian coast and in any case during the Last Glacial Maximum the islet was certainly connected to the mainland (Micalleff et al., 2013), followed in the Holocene by a rapid sea-level rise (Spampinato et al., 2011), but also in classical times (the Greek period) the sea level was at least 1 meter lower than at present (ScicchitANo et al., 2008). Therefore, it can be assumed that the islet had in the past a larger surface are and was less distant from the Sicilian coast. This probably allowed a human presence even if discontinuous, that ceased almost completely in the second half of the twentieth century. Due to its geomorphological shape, the environmental factors permitted colonisation by halophytes and psammophytes almost exclusively. The establishment of the nitrophilous component of the flora is due to the past human impact; this component currently is still well represented also in relation to the large population of gulls. The only alien species is Opuntia ficus-indica, which shows a limited ability to spread and thus its eradication should be pretty simple.

The results of this work will support the managing body of Vendicari Nature Reserve for conservation activities of the flora and fauna. Worth mentioning is the long lasting prohibition of landing on this islet, which has allowed the maintenance of an interesting flora and fauna, above all the seasonal establishment of Audouin's Gull, which finds here the special conditions of habitat and isolation that allow it to nest, making this unique site in Sicily. 


\section{ACKNOWLEDGEMENTS}

We thank the managing body of Vendicari Nature Reserve (Dipartimento Regionale Sviluppo Rurale e Territoriale - Province Unit of Syracuse) for funding the study and in particular Filadelfo Brogna, manager of the Nature Reserves in Syracuse PU and Giancarlo Perrotta, manager of the Germplasm Center of Vendicari, for the logistic support provided. Special thanks go to Salvatore Pasta, botanist, for pushing us to take our data out of the drawer and to the anonymous referees whose observations improved the quality of the final version of the manuscript.

Received June 1, 2016

\section{REFERENCES}

ALbo, G., 1959: La flora e la vegetazione delle isole intorno al Capo Pachino [The flora and vegetation of the islands around Cape Pachino]. Boll. Ist. Bot. Univ. Catania, serie 2, 1, 88-108.

Arrigoni, P.V. \& Bocchieri, E., 1996: Caratteri fitogeografici delle piccole isole circumsarde [Phytogeographical characteristics of the small circum-Sardinian islands]. Biogeographia 18, 63-90.

Bartolo, G., Brullo, S. \& Marcenò, C., 1982: La vegetazione costiera della Sicilia sud-orientale. Contributo alla interpretazione delle fasce di vegetazione delle coste mediterranee [The coastal vegetation of south-eastern Sicily. Contribution to the interpretation of the vegetation belts of the Mediterranean coasts]. C.N.R. AQ/1/226, pp.49.

Bazan, G., Marino, P., Guarino, R., Domina, G. \& Schicchi, R., 2015: Bioclimatology and vegetation series in Sicily: a geostatistical approach. Ann. Bot. Fennici 52, 1-18.

Biondi, E. \& Blasi, C. (ed.), 2010: Manuale italiano di interpretazione degli habitat della Direttiva 92/43 CEE [Italian interpretation manual of habitats of Directive 92/43 EEC]. Ministero dell' Ambiente. http:// vnr.unipg.it/habitat/index.jsp, (last accessed May 12, 2016).

Biondi, E., Blasi, C., Allegrrezza, M., Anzellotti, I., Azzella, M. M., Carli, E., Casavecchia, S., Copiz, R., Del Vico, E., Facioni, L., Galdenzi, D., Gasparri, R., Lasen, C., Pesaresi, S., Poldini, L., Sburlino, G., Taffetani, F., Vagge, I., Zitti, S. \& Zivkovic, L., 2014: Plant communities of Italy: The Vegetation Prodrome. Plant Biosyst., 148(4), 728-814.

Biondi, E., Casavecchia, S. \& Guerra, V., 2006: Analysis of vegetation diversity in relation to the geomorphological characteristics in the Salento coasts (Apulia - Italy). Fitosociologia 43(1), 25-38.

BogdAnović, S. \& Mitić, B., 2003: The flora of the volcanic island of Brusnik (central Dalmatia, Croatia). Acta Bot. Croat. 62(2), 103-113.

Braun-Blanquet, J., 1964: Pflanzensoziologie. Grundzüge der Vegetationskunde [Plant sociology. Basic course of Vegetation Science]. 3. New York, USA: Springer-Verlag.

Brullo, C., Minissale, P., Sciandrello, S. \& Spampinato, G., 2011a: Phytogeographic survey on the endemic vascular flora of the Hyblaean territory (SE Sicily, Italy). Acta Bot. Gallica 158(4), 617-631.

Brullo, C., Brullo, S., Giusso Del Galdo, G. P. \& Sciandrello, S., 2011b: Taxonomical, Morphological and Geobotanical considerations on Seseli tortuosum L. (Apiaceae) from Sicily. Phyton 51(2), 201-210.

Brullo, S., Fagotto, F. \& Marcenò, C., 1980: Carta della vegetazione di Vendicari [Map of the Vendicari vegetation]. Collana del programma finalizzato alla »Promozione della qualità dell'ambiente«. C. N. R., AQ/1/37-40: 25-41.

Brullo, S., Grillo, M. \& Guglielmo, A., 1998: Considerazioni fitogeografiche sulla flora iblea [Phytogeographical considerations about the Hyblaean flora]. Boll. Acc. Gioenia Sci. Nat. Catania 29(352), 45-111.

Brullo, S., Scelsi, F. \& Siracusa, G., 1994: Contributo alla conoscenza della vegetazione terofitica della Sicilia occidentale [Contribution to the knowledge of therophytic vegetation of western Sicily]. Boll. Acc. Gioenia Sci. Nat. Catania 27(346), 341-365.

BRULLO, S. \& SCELSI, F., 1998: Contributo alla conoscenza della vegetazione terofitica costiera del territorio ibleo [Contribution to the knowledge of the coastal therophytic vegetation of the Hyblaean territory]. Atti del Convegno »Flora e Vegetazione degli Iblei«. Boll. Acc. Gioenia Sci. Nat. Catania 29(352), 151168. 
Brullo, S., Minissale, P., Giusso Del Galdo, G., Siracusa, G. \& Spampinato, G., 2002: Considerazioni sintassonomiche e fitogeografiche sulla vegetazione della Sicilia [Syntaxonomic and phytogeographic considerations about the vegetation of Sicily]. Boll. Acc. Gioenia Sci. Nat. Catania 35(361), 325-359.

Davies, C. E., Moss, D., Hill M. O., 2004: EUNIS Habitat Classification Revised. European Environmental Agency.

Devillers, P., Devillers-Terschuren, J. \& Ledan, J-P., 1991: Corine Biotopes manual. vol. 2. Habitats of the European Community. Office for Official Publications of the European Communities, Luxembourg.

Dewey, J.F., Helman, M.L., Turco, E., Hutton, D.H.W. \& Knott, S.D., 1989: Kinematics of the Western Mediterranean. In: CowArd, M.P., et al. (eds.), Alpine tectonics. Geol. Soc. London Spec. Publ. 45, 265-283.EuropeAn commission, 2013: Interpretation Manual of European Union Habitat. EUR 28.

FIORI, A., 1923-1929: Nuova flora analitica d'Italia [New analytical flora of Italy]. Firenze.

Fois, M., Fenu, G. \& BacchettA, G., 2016: Global analyses underrate part of the story: finding applicable results for the conservation planning of small Sardinian islets flora. Biodivers. Conserv. 25(6), 10911106.

Giardina, G., Raimondo, F.M. \& Spadaro, V., 2007: A catalogue of plants growing in Sicily. Bocconea 20, 5-582.

Gracia, F. J., Geremia, F., Privitera, S. \& Amore, C., 2014: The probable karst origin and evolution of the Vendicari coastal lake system (SE Sicily, Italy). Acta Carsol. 43 (2-3), 215-228.

Houel, J., 1785: Voyage pittoresque des isles de Sicile, de Malte et de Lipari, où l'on traite des Antiquités qui s'y trouvent encore; des principaux Phénomènes que la Nature y offre; du Costume des Habitans, \& de quelques Usages [A picturesque tour of the islands of Sicily, Malta, and Lipari, where the Antiquities still in existence are treated; of the principal Phenomena which Nature offers; of the Costume of the Habitants, and of some Usages]. Vol. III. Paris.

Ientile, R., Linares, A. \& Brogna, F. 2016: First breeding colony of Audouin's Gull Larus audouinii in Sicily, characteristics and its origin. Avocetta 40, 71-76.

Iliadou, E., Panitsa, M., Raus, T. \& Dimopoulos, P., 2014: Flora and factors affecting species diversity in the islet groups of the protected »Natura 2000« sites of the Amvrakikos Gulf and Mesolongi Lagoon (Ionian area, Greece). Willdenowia 44, 439-450.

Jasprica, N., Dolina, K. \& Milović, M., 2015: Plant taxa and communities on three islets in south Croatia, NE Mediterranean. Nat. Croat. 24(2), 191-213.

Lentini, F., Catalano, S., Carbone, S., 1996: The external thrust system in Southern Italy: a target for petroleum exploration. Pet. Geosci. 2, 333-342.

Lo Cascio, P. \& Pasta, S., 2012: Lampione, a paradigmatic case of Mediterranean island biodiversity. Biodivers. J. 3, 311-330.

Malandrino, G., 2010: Presenza e attività umana a Vendicari [Human presence and activities in Vendicari]. In: Petralia A. (ed.) Atti del convegno »L'area protetta di Vendicari« - Case Cittadella Vendicari, October 25-26, 2008. p. 323-340.

Marcenò, C. \& Romano, S., 2010: La vegetazione psammofila della Sicilia settentrionale [The psammophilous vegetation of northern Sicily]. Inform. Bot. Ital. 42(1), 91-98.

Micallef, A., Foglini, F., Le Bas, T., Angeletti, L., Maselli, V., Pasuto, A. \& Taviani, M. 2013: The submerged paleolandscape of the Maltese Islands: Morphology, evolution and relation to quaternary environmental change. Marine Geology 335, 129-147.

Minissale, P. \& Sciandrello, S., 2010: Flora e vegetazione terrestre della Riserva Naturale di Vendicari (Sicilia sud-orientale) [Terrestrial flora and vegetation of the Vendicari Nature Reserve (south-eastern Sicily)]. In: Petralia A. (ed.) Atti del convegno »L'area protetta di Vendicari« - Case Cittadella Vendicari, October 25-26, 2008. p. 131-194.

PAndžA, M., 2002: Flora of the small islands of Murter. Nat. Croat. 11(1), 77-101.

PAndžA, M. \& Milović, M., 2015: Flora of the Veliki Lagan and Mali Lagan islets (Dugi otok island, Croatia). Nat. Croat. 24(2), 215-222.

Panitsa, M, Tzanoudakis, D, Triantis, K.A. \& Sfenthourakis, S., 2006: Patterns of species richness on very small islands: the plants of the Aegean archipelago. J. Biogeogr. 33(7), 1223-1234.

Pasta, S., Sciberras, A., Sciberras, J. \& Scuderi, L., 2014: Analysis of the vascular flora of four satellite islets of the Egadi Archipelago (W Sicily), with some notes on their vegetation and fauna. Biodivers. J. 5(1), 39-54. 
Pirrotta, C. \& Barbano, M. B., 2011: Analysis of deformation structures in Pliocene and Quaternary deposits of the Hyblean Plateaux (South-Eastern Sicily.) Tectonophysics 499, 41-53.

PignatTi, S., 1982: Flora d'Italia [Flora of Italy]. 1-3. Edagricole, Bologna.

Sciandrello, S., Minissale, P. \& Sturiale, G., 2017: Plant communities supported by the geological setting: the case history of the Isole dei Ciclopi (Etna, east Sicily). Lazaroa 38 (in press).

Scicchitano, G., Antonioli, F., Castagnino Berlinghieri, E. F., Dutton, A. \& Monaco, C., 2008: Submerged archaeological sites along the Ionian coast of southeastern Sicily (Italy) and implications for the Holocene relative sea-level change. Quatern. Res. 70, 26-39.

Spampinato, C.R., Costa, B., Di Stefano, A., Monaco, C. \& Scicchitano, G., 2011: The contribution of tectonics to relative sea-level change during the Holocene in coastal south-eastern Sicily: new data from boreholes. Quatern. Int. 232, 214-227.

Zampino, D., Duro, A., Piccione, V. \& Scalia, C., 1997:- Termoudogrammi secondo Walter \& Lieth [Thermoudogram according to Walter \& Lieth]. Atti $5^{\circ}$ Workshop Progetto Strategico »Clima Ambiente e Territorio nel Mezzogiorno« 2, 7-54. 